\title{
Démontrer la capacité du Comité consultatif national de l'immunisation à réagir rapidement aux signaux de surveillance des vaccins après leur mise sur le marché : l'expérience du Canada avec le vaccin antigrippal vivant atténué
}

\author{
Linlu Zhao ${ }^{1 *}$, Kelsey Young ${ }^{1}$, Althea House ${ }^{1}$, Rob Stirling ${ }^{1}$, Matthew Tunis ${ }^{1}$
}

\section{Résumé}

Au cours des dernières années, I'utilisation recommandée du vaccin vivant atténué contre l'influenza (VVAl) pour les enfants a évolué aux États-Unis en réponse aux preuves d'une diminution potentielle de l'efficacité du WVI basées sur la surveillance post-commercialisation. Ces problèmes n'ont pas été observés au Canada ou ailleurs; par conséquent, les recommandations du Comité consultatif national de l'immunisation (CCNI) du Canada et du Advisory Committee on Immunization Practices (ACIP) des États-Unis sur l'opportunité d'utiliser le WAI ont différé pour deux saisons grippales (2016-2017 et 2017-2018).

Cette rétrospective décrit comment le $\mathrm{CCNI}$ est parvenu à ses recommandations en réponse aux signaux post-commercialisation d'une baisse de performance des WAI provenant des États-Unis en 2013-2014 et à nouveau en 2015-2016. L'expérience du CCNI avec le VVAI marque la première fois au Canada où une recommandation préférentielle sur l'utilisation d'un vaccin antigrippal dans un programme d'immunisation systématique a été renversée. Cette expérience souligne l'importance d'une surveillance continue des vaccins après leur mise sur le marché, d'une collaboration internationale et d'une prise en compte attentive du contexte local pour étayer les recommandations relatives aux vaccins. La capacité du CCNI à réagir rapidement aux signaux de performance des vaccins après leur mise sur le marché facilitera la réaction à des signaux similaires de surveillance après la mise sur le marché des vaccins contre la maladie à coronavirus 2019 (COVID-19).
Cette oeuvre est mise à la disposition selon les termes de la licence internationale Creative Commons Attribution 4.0

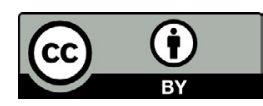

Affiliation

1 Agence de la santé publique du Canada

*Correspondance :

linlu.zhao@canada.ca

Citation proposée : Zhao L, Young K, House A, Stirling R, Tunis M. Démontrer la capacité du Comité consultatif national de l'immunisation à réagir rapidement aux signaux de surveillance des vaccins après leur mise sur le marché : l'expérience du Canada avec le vaccin antigrippal vivant atténué. Relevé des maladies transmissibles au Canada 2021;47(5/6):306-11. https://doi.org/10.14745/ccdr.v47i56a06f

Mots-clés : grippe, VVAl, vaporisateur nasal, surveillance post-marché, efficacité du vaccin

\section{Introduction}

L'Organisation mondiale de la Santé recommande que chaque pays dispose d'un Groupe technique consultatif national pour la vaccination composé d'experts chargés de formuler des recommandations indépendantes sur les vaccins, fondées sur des données probantes $(1,2)$. Le Comité consultatif national de l'immunisation (CCNI) du Canada, un organisme consultatif externe de l'Agence de la santé publique du Canada (l'Agence), est l'un des plus anciens Groupe technique consultatif national pour la vaccination au monde. Depuis plus de 50 ans, le CCNI fournit de manière continue et opportune des conseils d'experts et fondés sur des preuves concernant l'utilisation des vaccins pour protéger les Canadiens.

Les recommandations du CCNI sont élaborées à l'aide d'un processus fondé sur des données probantes, qui implique globalement les étapes séquentielles de recherche de preuves, de synthèse et de traduction en recommandations (3). Les éléments déclencheurs de l'élaboration des lignes directrices du CCNI comprennent, sans s'y limiter, I'autorisation de nouveaux vaccins sur le marché canadien; les modifications des 
formulation trivalente du VVAl a été remplacée par la formulation quadrivalente au Canada à partir de la saison grippale 20142015.

En 2011, le CCNI a fait une recommandation préférentielle pour l'utilisation du VVAl trivalent par rapport au VAI trivalent pour les enfants et les adolescents âgés de 2 à 17 ans pour la saison 2011-2012 (les vaccins antigrippaux quadrivalents n'étaient pas disponibles au Canada à ce moment-là) (4). Cette décision a été prise sur la base de données d'efficacité favorable issue d'essais cliniques et de données de sécurité post-commercialisation, avec la reconnaissance de preuves plus solides d'une efficacité supérieure chez les jeunes enfants (moins de six ans) que chez les enfants plus âgés. Sur la base des données d'efficacité, en juin 2014, l'Advisory Committee on Immunization Practices (ACIP), I'homologue américain du CCNI, a recommandé l'utilisation préférentielle du WVAI quadrivalent par rapport au VAl trivalent ou quadrivalent pour la saison 2014-2015 chez les enfants de 2 à 8 ans. La limite d'âge supérieure de la recommandation de l'ACIP a été choisie en fonction de la cohérence programmatique (i.e. qu'aux ÉtatsUnis, l'âge de huit ans est également la limite d'âge supérieure pour l'administration de deux doses de vaccin contre la grippe à un enfant non vacciné auparavant) (5). Ces recommandations d'utilisation préférentielle des VVAI formulées par le CCNI et I'ACIP étaient en place lorsque les résultats de la réduction de I'efficacité des VVAl contre la grippe A (H1N1) au cours de la saison 2013-2014 aux États-Unis ont été révélés $(4,5)$.

La figure 1 présente un résumé visuel des étapes de I'élaboration des recommandations sur l'utilisation des VVAI au Canada et aux États-Unis. Le tableau 1 présente un résumé verbal de ces événements.

\section{Figure 1 : Étapes des recommandations sur l'utilisation des VVAl au Canada et aux États-Unis}

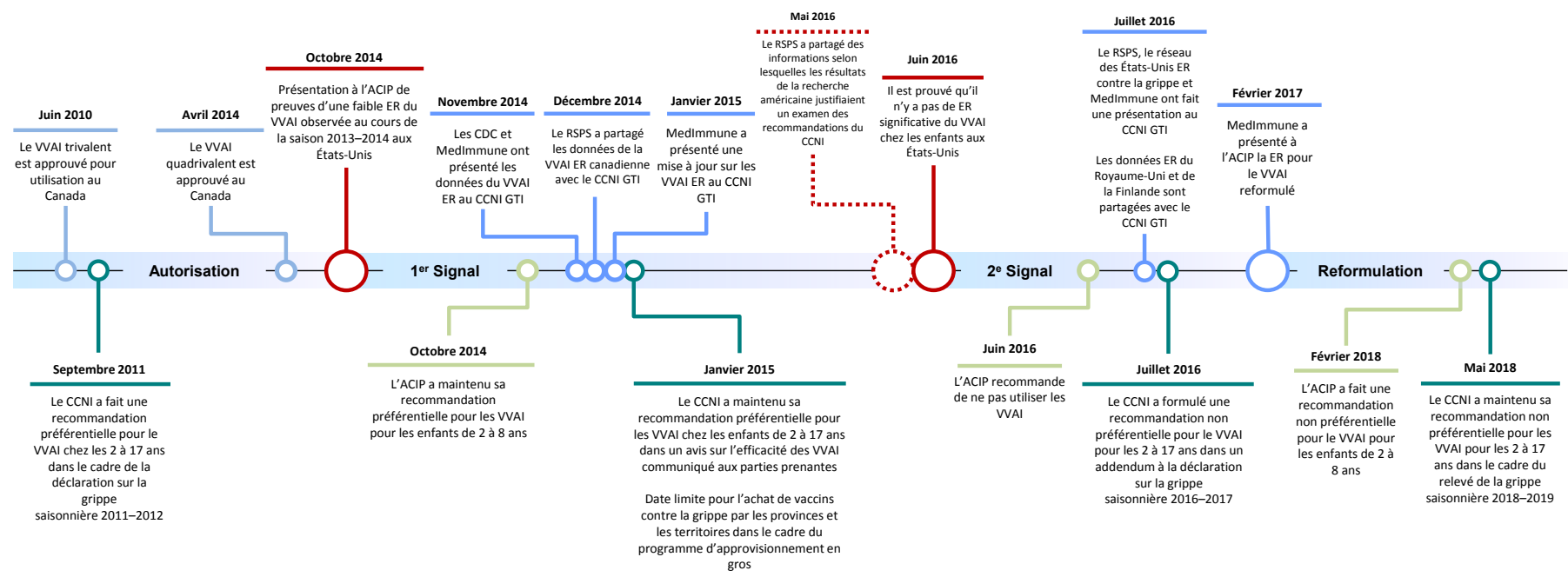

Abréviations : ACIP, Advisory Committee on Immunization Practices; CCNI, Comité consultatif national de l'immunisation; CDC, Centers for Disease Control and Prevention des États-Unis; ER, Efficacité réelle du vaccin; GTI, Groupe de travail sur l'Influenza; RSPS, Le Réseau de surveillance des praticiens sentinelles; VVAl, vaccin vivant atténué contre l'influenza 
Tableau 1 : Chronologie des réponses du CCNI aux signaux de performance du VVAl en provenance des États-Unis

\begin{tabular}{|c|c|}
\hline Date & Réponse \\
\hline Premier signal & $\begin{array}{l}\text { Le } 29 \text { octobre 2014, des preuves de la faible } \\
\text { efficacité des VVAl pour la saison grippale } \\
2013-2014 \text { aux États-Unis ont été présentées à } \\
\text { l'ACIP. }\end{array}$ \\
\hline $\begin{array}{l}18 \text { novembre } \\
2014\end{array}$ & $\begin{array}{l}\text { Les données sur l'efficacité du WVAl aux États-Unis } \\
\text { pour la saison } 2013-2014 \text { ont été présentées lors } \\
\text { d'une réunion conjointe du CCNI, du GTI du CCNI et } \\
\text { du CIC (représentant les programmes d'immunisation } \\
\text { provinciaux et territoriaux). }\end{array}$ \\
\hline $\begin{array}{l}\text { Fin novembre } \\
\text { à décembre } \\
2014\end{array}$ & $\begin{array}{l}\text { Le RSPS du Canada a partagé avec le CCNI les } \\
\text { données sur l'efficacité des WAI pour la saison } \\
\text { 2013-2014. Sur la base des données disponibles, le } \\
\text { CCNI a conclu qu'aucun changement ne sera apporté } \\
\text { à la recommandation sur l'utilisation des VVAI pour la } \\
\text { saison 2015-2016. }\end{array}$ \\
\hline 26 janvier 2015 & $\begin{array}{l}\text { La position du CCNI sur l'utilisation des VVAl en } \\
\text { réponse au premier signal a été distribuée aux } \\
\text { intervenants provinciaux et territoriaux. }\end{array}$ \\
\hline $\begin{array}{l}\text { Deuxième } \\
\text { signal }\end{array}$ & $\begin{array}{l}\text { Le } 22 \text { juin } 2016 \text {, I'ACIP a recommandé de ne pas } \\
\text { utiliser les VVAl pour la saison } 2016-2017 \text { en se } \\
\text { basant sur des preuves de la faible efficacité des } \\
\text { WAI pour deux saisons de grippe A (H1N1)pdm09 } \\
\text { prédominantes (2013-2014 et 2015-2016) aux } \\
\text { États-Unis. }\end{array}$ \\
\hline 4 juillet 2016 & $\begin{array}{l}\text { L'Agence a organisé une téléconférence avec le } \\
\text { CIC pour discuter du signal d'efficacité des WVAl en } \\
\text { provenance des États-Unis et des plans du CCNI } \\
\text { pour examiner les preuves disponibles. Le secrétariat } \\
\text { du CCNI à l'Agence a commencé à synthétiser les } \\
\text { données disponibles sur l'efficacité des WVAl après } \\
2009 \text {. }\end{array}$ \\
\hline 19 juillet 2016 & $\begin{array}{l}\text { Les données sur l'efficacité des VVAl après 2009, } \\
\text { y compris les données du Canada, des États-Unis } \\
\text { et d'autres juridictions pour la saison grippale } \\
\text { 2015-2016, ont été présentées au GTI du CCNI pour } \\
\text { délibération. Sur la base des preuves disponibles, le } \\
\text { GTI a proposé un changement de recommandation à } \\
\text { l'attention du CCNI. }\end{array}$ \\
\hline 26 juillet 2016 & $\begin{array}{l}\text { Le CCNI a conclu que les données disponibles } \\
\text { ne permettaient pas de recommander I'utilisation } \\
\text { préférentielle du WVAl par rapport au VAl, mais que } \\
\text { le WAI restait une option de vaccination contre la } \\
\text { grippe pour les enfants. }\end{array}$ \\
\hline 29 juillet 2016 & $\begin{array}{l}\text { La position du CCNI sur l'utilisation des VVAl en } \\
\text { réponse au deuxième signal a été distribuée aux } \\
\text { intervenants provinciaux et territoriaux. }\end{array}$ \\
\hline
\end{tabular}

Abréviations : ACIP, Comité consultatif sur les pratiques d'immunisation; Agence, Agence de la santé publique du Canada; $\mathrm{CIC}$, Comité canadien d'immunisation; CCNI, Comité consultatif national de l'immunisation; GTI, Groupe de travail sur I'Influenza; RSPS, Le Réseau de surveillance des praticiens sentinelles; VAl, vaccin antigrippal inactivé; VAI, vaccin vivant atténué contre l'influenza

\section{Premier élément déclencheur d'une éventuelle révision par le CCNI des orientations relatives aux VVAl}

En octobre 2014, quelques mois après que l'ACIP ait formulé sa recommandation préférentielle, des études de surveillance post-commercialisation ont trouvé des preuves d'une efficacité faible à nulle des VAI contre la grippe A (H1N1) chez les enfants et adolescents américains âgés de 2 à 17 ans au cours de la saison 2013-2014; ces preuves ont été présentées à l'ACIP (6).

La faible performance des WVI observée aux États-Unis a conduit le $\mathrm{CCNI}$ à entreprendre un examen rapide des données probantes afin de déterminer s'il était nécessaire de réviser sa recommandation sur la façon dont les WVAl devraient continuer à être utilisés au Canada. L'examen des données probantes devait être rapide pour éclairer les décisions d'approvisionnement des provinces et des territoires qui seraient prises au début de 2015 pour la saison grippale 2015-2016. En novembre 2014, des conférenciers invités des Centre de contrôle et de prévention des maladies américains(CDC) et de Medlmmune, le fabricant de VVAl, avaient présenté leurs données sur l'efficacité des VVAl américains au $\mathrm{CCNI}$ et à son groupe de travail sur la grippe (GTI). À l'époque, Medlmmune a proposé que l'efficacité réduite contre la grippe $A(H 1 N 1)$ observée aux États-Unis pouvait être due à la vulnérabilité à la dégradation thermique de la souche A/California/7/2009(H1N1)pdm09 présente dans le vaccin, qui a pu se produire pendant la distribution du vaccin. En décembre 2014, le Réseau de surveillance des praticiens sentinelles du Canada a partagé avec le CCNI des données canadiennes non publiées sur l'efficacité du VVAl pour la saison 2013-2014, qui montraient que l'efficacité du WVAl était similaire à celle du VAI, ce qui différait des données américaines.

Après avoir examiné ces données d'efficacité de la saison 2013-2014, le CCNI a publié une déclaration d'opinion fin janvier 2015, à temps pour informer les décisions d'approvisionnement des provinces et des territoires pour la prochaine saison 2015-2016. La déclaration d'opinion indiquait qu'aucun changement ne serait apporté à la recommandation préférentielle du CCNI sur I'utilisation des VVAI (7). La décision du CCNI a également tenu compte de facteurs autres que l'efficacité réelle du vaccin, notamment les différentes formulations de WAI utilisées aux États-Unis (quadrivalent) et au Canada (trivalent) en 2013-2014; les différences dans la distribution du vaccin à température contrôlée entre les deux pays; et le fait que la faible efficacité du WVAl observée dans les études américaines pour la saison 2013-2014 n'a pas été constatée dans les données canadiennes sur l'efficacité du VVAI après la mise en marché du Réseau de surveillance des praticiens sentinelles pour la même saison (8). En revanche, en février 2015, I'ACIP est revenu sur sa recommandation d'utilisation préférentielle du WVAl pour indiquer que le WAI ou le VAI étaient des options vaccinales appropriées chez les enfants âgés de 2 à 8 ans pour la saison 2015-2016 (9).

À la suite des études de stabilité thermique menées par le fabricant, le composant $\mathrm{A}(\mathrm{H} 1 \mathrm{~N} 1) \mathrm{pdm09}$ du WAl a été changé pour la saison 2014-2015, passant de la souche A/California/7/2009 à la souche A/Bolivia/559/2013, antigéniquement similaire et plus stable à la chaleur. La question de savoir si le changement de souche pour le VVAl a amélioré 
ses performances pour la saison grippale 2014-2015 par rapport au vaccin inactivé n'a pas été concluante, la saison ayant été dominée par des virus $A(H 3 N 2)$ à dérive antigénique. Le VVAI et le VAl ont tous deux obtenu des résultats médiocres pour la saison 2014-2015; une majorité d'études n'ont trouvé aucune preuve que l'un ou l'autre type de vaccin protégeait contre toute forme de grippe et contre la grippe A (H3N2) (10).

\section{Deuxième élément déclencheur d'un éventuel examen des orientations par le CCNI}

Les études de surveillance post-commercialisation achevées aux États-Unis à la fin de la saison 2015-2016 ont à nouveau révélé une faible efficacité vaccinale pour le VVAl, mais pas pour le VAl, contre la grippe $\mathrm{A}$ (H1N1) chez les enfants et les adolescents âgés de 2 à 17 ans (11). Le 22 juin 2016, I'ACIP a recommandé de ne pas utiliser les WAI pour la saison 2016-2017. La décision de l'ACIP a été motivée par la réduction de l'efficacité observée pour les VVAl contre le A (H1N1) aux États-Unis au cours des saisons 2013-2014 et 2015-2016 où circulaient principalement des virus de type $A(H 1 N 1) p d m 09(12,13)$. Cette décision a suscité une attention et un intérêt importants de la part des parties prenantes canadiennes.

Les nouvelles données américaines sur le VVAl et la recommandation de I'ACIP contre l'utilisation du VVAI aux États-Unis ont suscité des inquiétudes quant à l'utilisation du VAl au Canada par les programmes d'immunisation provinciaux et territoriaux, qui s'étaient déjà procuré du VVAl. Tous ces facteurs ont été des éléments déclencheurs pour que le CCNI revoie une fois de plus ses directives sur l'utilisation des VVAI au Canada.

Le défi du CCNI pour répondre à ce déclencheur a été la contrainte de temps pour fournir une décision très rapide, fondée sur des données probantes, concernant l'utilisation du WAI déjà acheté, à quelques mois du début des campagnes de vaccination provinciales et territoriales (généralement en octobre) pour la saison à venir. Le 4 juillet 2016, l'Agence a tenu une réunion avec des intervenants des programmes d'immunisation provinciaux et territoriaux afin de les informer des nouvelles données sur l'efficacité des WVAl provenant des États-Unis et de les informer des activités prévues par le CCNI pour examiner d'autres données canadiennes et internationales sur l'efficacité des WVAl afin d'éclairer l'examen par le CCNI de ses recommandations sur les VVAI.

Le 19 juillet 2016, le GTI du CCNI a examiné des données non publiées de surveillance post-commercialisation sur l'efficacité des VVAI pour la saison grippale 2015-2016 provenant de six sources : Le Réseau de surveillance des praticiens sentinelles du Canada, le réseau américain d'efficacité réelle des vaccins contre la grippe (US Flu VE Network), le ministère américain de la Défense, le réseau britannique d'efficacité des vaccins contre la grippe, I'Institut national de la santé et du bien-être de Finlande et Medlmmune. Le GTI a également examiné les données publiées et non publiées sur la pandémie de 2009 afin d'évaluer la tendance de l'efficacité des VVAl au cours des saisons grippales depuis que la souche pandémique $A(H 1 N 1) p d m 09$ a remplacé les souches de grippe $A(H 1 N 1)$ prépandémiques. Il s'agit notamment des résultats de deux essais canadiens contrôlés randomisés en grappes qui n'ont pas constaté de réduction de l'efficacité des VVAl pour la saison 2013-2014 au Canada $(14,15)$, ce qui concorde avec les résultats du Réseau de surveillance des praticiens sentinelles pour cette saison.

À la suite de l'examen des données disponibles, qui ont montré que les VVAl offraient une protection contre la grippe comparable à celle offerte par les VAl et de discussions avec diverses juridictions, le GTI a recommandé au CCNI de modifier ses recommandations sur l'utilisation des VVAl. Le 26 juillet 2016, sur la base de l'avis du GTI et après avoir examiné toutes les données disponibles, le $\mathrm{CCNI}$ a conclu que les données disponibles ne permettaient plus de recommander l'utilisation préférentielle du VVAI par rapport au VAI, mais que le VVAI restait une option pour la vaccination annuelle contre la grippe des enfants âgés de 2 à 17 ans (6). Bien que les données américaines montrant que l'efficacité des VVAl était comparable ou inférieure à celle des VAI aient contribué à la recommandation révisée et non préférentielle du CCNI sur l'utilisation des VVAl, la réduction de l'efficacité observée aux États-Unis pour la saison 2015-2016 n'a encore pas été observée au Canada ou dans d'autres pays qui ont étudié la question (10). La différence entre les données de performance des VVAl du Canada et d'autres juridictions internationales par rapport aux données des États-Unis a joué un rôle important dans la décision du CCNI de continuer à recommander l'utilisation des VVAl au Canada pour la saison 2016-2017 (16). La position officielle du CCNI a été communiquée aux parties prenantes fin juillet $2016(6,10,16)$. Les données sur l'efficacité à l'appui de la décision du CCNI ont été détaillées ailleurs (10).

\section{Épilogue}

La mauvaise performance du composant A (H1N1) du VVAI lors de la saison grippale 2015-2016 a été attribuée à la réduction de la capacité de réplication de la souche vaccinale A(H1N1)pdm09-like. Comme pour la saison 2013-2014, les différences dans la distribution des vaccins à température contrôlée entre le Canada et les États-Unis pourraient être un facteur de la performance différentielle du VVAI dans les deux pays pour la saison 2015-2016. En raison de la constatation d'une capacité de réplication réduite, la souche A(H1N1)pdm09-like de la formulation du vaccin 2015-2016 (A/Bolivia/559/2013) a été remplacée par une nouvelle souche (A/Slovenia/2903/2015) pour la saison 2017-2018 (13).

L'ACIP a maintenu sa recommandation contre l'utilisation des VAI pour la saison 2017-2018. Cependant, en février 2018, I'ACIP a voté pour rétablir le WVI comme option vaccinale pour la saison 2018-2019, sans donner de préférence au VVAI 
ou au VAI pour le groupe d'âge pédiatrique, sur la base de données fournie par le fabricant suggérant que la nouvelle souche $A(H 1 N 1) p d m 09-l i k e(A / S l o v e n i a / 2903 / 2015)$ a une meilleure aptitude à la réplication par rapport aux précédentes

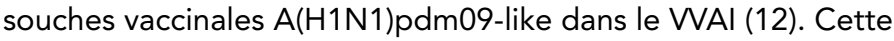
recommandation non préférentielle est maintenue par l'ACIP dans sa dernière déclaration pour la saison 2020-2021(17). La recommandation du CCNI concernant l'utilisation non préférentielle des VVAl chez les enfants et les adolescents âgés de 2 à 17 ans reste inchangée pour la saison grippale 2021-2022 (18).

\section{Discussion}

Le CCNI et l'ACIP sont parvenus à des conclusions différentes sur l'utilisation des WVI en réponse aux signaux de performance des VVA après leur commercialisation aux États-Unis pour les saisons 2013-2014 et 2015-2016, mais les recommandations divergentes des Groupe technique consultatif national pour la vaccination ne sont pas inhabituelles. Les Groupe technique consultatif national pour la vaccination tiennent compte d'une multitude de facteurs lorsqu'ils formulent leurs recommandations, notamment les caractéristiques des vaccins (efficacité potentielle, efficacité réelle, immunogénicité et sécurité), la charge de morbidité locale et les données sur l'efficacité réelle des vaccins. Sur la base de cette analyse multifactorielle complexe et de la pondération différente de ces facteurs dans leurs analyses, il n'est pas surprenant que les Groupe technique consultatif national pour la vaccination arrivent souvent à des conclusions différentes quant à la stratégie de vaccination qui répondra le mieux aux besoins de leur pays spécifique.

L'évaluation rapide des preuves et la prise de décision du CCNI en réponse aux faibles données sur l'efficacité du WVAl aux États-Unis ont été facilitées par plusieurs facteurs critiques. Le fait de pouvoir compter sur les évaluations de l'efficacité réelle du vaccin contre la grippe saisonnière effectuée par les réseaux canadiens établis de surveillance de la grippe a été un élément facilitateur. Le Réseau de surveillance des praticiens sentinelles du Canada a fourni une évaluation en temps quasi réel de ses données au CCNI et à l'Agence afin d'éclairer les délibérations du CCNI sur l'utilisation des WVI chez les enfants.

Le fait de disposer de données sur l'efficacité réelle du vaccin antigrippal canadien provenant d'études spécifiquement conçues et dotées d'une puissance appropriée pour comparer le VAI et le VAl, comme les essais cliniques randomisés en grappes qui ont corroboré les résultats de la surveillance du Réseau de surveillance des praticiens sentinelles de la saison 2013-2014, a également facilité les choses. (Le Réseau de surveillance des praticiens sentinelles n'a pas été conçu pour comparer l'efficacité de produits vaccinaux spécifiques).
Un troisième facilitateur a été la collaboration avec des partenaires internationaux et industriels. Le partage de l'information sur les signaux émergents et les données non publiées a été essentiel pour permettre au CCNI d'élaborer ses orientations sur la base des meilleures données disponibles dans ces situations où le temps est compté. Plusieurs facteurs critiques ont également contribué à relever avec succès le défi de la mise en application des données probantes d'un point de vue opérationnel. Il s'agit notamment d'avoir établi des processus pour faciliter l'engagement des parties prenantes et de mobiliser la capacité de pointe au sein de l'Agence pour fournir un soutien technique et logistique au CCNI.

\section{Conclusion}

La réponse du CCNI aux signaux de performance des VAI après leur mise sur le marché aux États-Unis est un exemple de sa capacité à réagir rapidement et de manière globale au paysage dynamique de la recherche internationale sur les vaccins et de l'épidémiologie des maladies infectieuses. Ces réponses soulignent l'importance d'établir et d'exploiter les canaux existants pour le partage de renseignements et l'échange de connaissances avec les producteurs, les partenaires et les utilisateurs de données probantes, ainsi que l'importance de tenir compte des données probantes provenant de sources multiples et du contexte local pour éclairer la prise de décision. L'expérience et la capacité du CCNI à réagir rapidement aux signaux de performance des vaccins après leur mise sur le marché faciliteront la réaction à des signaux similaires de surveillance après la mise sur le marché des vaccins contre la maladie à coronavirus 2019 (COVID-19).

\section{Déclaration des auteurs}

L. Z. - Conceptualisation, rédaction de la version originale, rédaction de la révision et de l'édition

K. Y. - Conceptualisation, rédaction-révision et édition

A. H. - Conceptualisation, rédaction-révision et édition

R. S. - Conceptualisation, rédaction-révision et édition

M. T. - Conceptualisation, rédaction-révision et édition

\section{Intérêts concurrents}

Aucun.

\section{Financement}

Ce travail a été soutenu par l'Agence de la santé publique du Canada. 


\section{Références}

1. Adjagba A, MacDonald NE, Ortega-Pérez I, Duclos P; 2016 Global NITAG Network Meeting Participants. Strengthening and sustainability of national immunization technical advisory groups (NITAGs) globally: lessons and recommendations from the founding meeting of the global NITAG network. Vaccine 2017;35(23):3007-11. DOI PubMed

2. Duclos P. National Immunization Technical Advisory Groups (NITAGs): guidance for their establishment and strengthening. Vaccine 2010;28 Suppl 1:A18-25.

DOI PubMed

3. Comité consultatif national de l'immunisation (CCNI). Recommandations pour l'immunisation fondées sur des données probantes - Méthodes du Comité consultatif national de l'immunisation. Relevé des maladies transmissibles au Canada 2009;35(ACS-1):1-10. https://www.canada.ca/content/ $\mathrm{dam} / \mathrm{phac}$-aspc/migration/phac-aspc/publicat/ccdr-rmtc/09pdf/ ccdr-rmtc-vol-35-acs-dcc-1.pdf

4. Membres du Groupe de travail sur l'influenza. Une déclaration d'un comité consultatif (DCC) Comité consultatif national de I'immunisation (CCNI). Recommandations relatives à I'utilisation du vaccin antigrippal vivant atténué $\left(\right.$ FluMist $\left.^{\circledR}\right)$ Déclaration complémentaire sur la vaccination antigrippale pour la saison 2011-2012. Relevé des maladies transmissibles au Canada 2011;37(ACS-7):1-86. DOI

5. Grohskopf LA, Olsen SJ, Sokolow LZ, Bresee JS, Cox NJ, Broder KR, Karron RA, Walter EB; Centers for Disease Control and Prevention. Prevention and control of seasonal influenza with vaccines: recommendations of the Advisory Committee on Immunization Practices (ACIP) -- United States, 2014-15 influenza season. MMWR Morb Mortal Wkly Rep 2014;63(32):691-7. PubMed

6. Comité consultatif national de l'immunisation (CCNI). Chapitre sur la grippe du Guide canadien d'immunisation et Déclaration sur la vaccination antigrippale pour la saison 2016-2017 : ADDENDA - Utilisation du WVAl chez les enfants et les adolescents. Ottawa (ON) : ASPC; 2016 (modifié 2018-02-21; accédé 2018-06-20). https://www.canada. $\mathrm{ca} / \mathrm{fr} /$ sante-publique/services/immunisation/comite-consultatifnational-immunisation-ccni/chapitre-grippe-guide-canadienimmunisation-declaration-vaccination-antigrippale-saison-20162017-addenda-utilisation-vvao-chez-enfants-adolescents.html

7. Comité consultatif national de l'immunisation (CCNI). L'avis du Comité consultatif national de l'immunisation concernant l'efficacité des vaccins antigrippaux vivants atténués chez les jeunes enfants. Ottawa (ON) : ASPC; 2015 (modifié 2015-02-24; accédé 2018-06-20). https://www.canada. $\mathrm{ca} / \mathrm{fr} /$ sante-publique/services/publications/vie-saine/avis-comiteacute-consultatif-national-immunisation-concernant-efficaciteacute-vaccins-antigrippaux-vivants-att-eacute-nu-eacute-chezjeunes-enfants.html

8. Une déclaration d'un comité consultatif (DCC) - Comité consultatif national de l'immunisation (CCNI) : Chapitre sur la grippe du Guide canadien d'immunisation et Déclaration sur la vaccination antigrippale pour la saison 2015-2016. Ottawa (ON) : ASPC; 2015 (modifié 2016-03-08; accédé 2018-12-07). https://www.canada.ca/fr/sante-publique/services/immunisation/ comite-consultatif-national-immunisation-ccni/declarationvaccination-antigrippale-saison-2015-2016.html
9. Grohskopf LA, Sokolow LZ, Olsen SJ, Bresee JS, Broder KR Karron RA. Prevention and Control of Influenza with Vaccines: Recommendations of the Advisory Committee on Immunization Practices, United States, 2015-16 Influenza Season. MMWR Morb Mortal Wkly Rep 2015;64(30):818-25. DOI PubMed

10. Vaudry W, Stirling R; Comité consultatif national de I'immunisation (CCNI). Sommaire de la Déclaration du CCNI sur la vaccination antigrippale pour la saison 2017-2018. Relevé des maladies transmissibles au Canada. 2017;43(5):106-14. DOI

11. Department of Health and Human Services Centers for Disease Control and Prevention CDC. Advisory Committee on Immunization Practices (ACIP): summary report. Atlanta (GA): US Centers for Disease Control and Prevention; 2016. https:// www.cdc.gov/vaccines/acip/meetings/downloads/min-archive/ min-2016-06.pdf

12. Grohskopf LA, Sokolow LZ, Broder KR, Olsen SJ, Karron RA, Jernigan DB, Bresee JS. Prevention and Control of Seasonal Influenza with Vaccines Recommendations of the Advisory Committee on Immunization Practices-United States, 2016-17 influenza season. MMWR Recomm Rep 2016;65(5):1-54. DOl PubMed

13. Grohskopf LA, Sokolow LZ, Fry AM, Walter EB, Jernigan DB. Update: ACIP recommendations for the use of quadrivalent live attenuated influenza vaccine (LAIV4) - United States, 2018-19 Influenza Season. MMWR Morb Mortal Wkly Rep 2018;67(22):643-5. DOI PubMed

14. Kwong JC, Pereira JA, Quach S, Pellizzari R, Dusome E, Russell ML, Hamid JS, Feinberg Y, Winter AL, Gubbay JB, Sirtonski B, Moher D, Sider D, Finkelstein M, Loeb M; Public Health Agency of Canada/Canadian Institutes of Health Research Influenza Research Network (PCIRN) Program Delivery and Evaluation Group. Randomized evaluation of live attenuated vs. inactivated influenza vaccines in schools (RELATIVES) cluster randomized trial: pilot results from a household surveillance study to assess direct and indirect protection from influenza vaccination. Vaccine 2015;33(38):4910-5. DOI PubMed

15. Loeb M, Russell ML, Manning V, Fonseca K, Earn DJ, Horsman G, Chokani K, Vooght M, Babiuk L, Schwartz L, Neupane B, Singh $P$, Walter SD, Pullenayegum E. Live attenuated versus inactivated influenza vaccine in Hutterite children: a cluster randomized blinded trial. Ann Intern Med 2016;165(9):617-24. DOI PubMed

16. Tam TW. Intranasal influenza vaccine: why does Canada have different recommendations from the USA on its use? Paediatr Child Health 2018;23(1):31-4. DOI PubMed

17. Grohskopf LA, Alyanak E, Broder KR, Blanton LH, Fry AM, Jernigan DB, Atmar RL. Prevention and control of seasonal influenza with vaccines: recommendations of the Advisory Committee on Immunization Practices United States, 2020-21 Influenza Season.

MMWR Recomm Rep 2020;69(8):1-24. DOI PubMed

18. Comité consultatif national de l'immunisation (CCNI). Une déclaration d'un comité consultatif (DCC) - Comité consultatif national de l'immunisation (CCNI) : Chapitre sur la grippe du Guide canadien d'immunisation et Déclaration sur la vaccination antigrippale pour la saison 2020-2021. Ottawa (ON) : ASPC; 2020 (modifié 2021-01-28; accédé 2021-05-04). https://www. canada.ca/fr/sante-publique/services/publications/vaccinsimmunisation/guide-canadien-immunisation-declarationvaccination-antigrippale-2020-2021.html 